\title{
Biosurveillance De La Qualite De L'air D'un Milieu Urbain Par Des Mousses Et Des Lichens. Cas De La Ville De Tlemcen (Algerie)
}

\author{
Berrayah Mohammed \\ Maatoug M'hamed \\ Azzaoui Mohamed \\ Diallo Oumou \\ Sidibé Alimata \\ Manaa Khadija Hadjar
}

Laboratoire d'Agro Biotechnologie et de Nutrition en Zones Semi Arides Faculté des Science de la Nature et de la vie,

Université Ibn Khaldoun Tiaret, Algérie

\begin{abstract}
The evaluation of the pollution intensity, the accumulation of pollutants in the atmosphere, by living organisms is a very effective environmental technique used for decades. The measure of the accumulation in these organisms allows meeting the direct difficulties in physical and chemical measures, often very complex and costly. By physiological and morphological characteristics, mosses and lichens are very effective to address this issue, including their capacity to accumulate contaminants in this case the trace metals such as lead. The objective of this work is to measure lead concentrations from road origin in different urban sites in Tlemcen city (Algeria) using two species of moss and lichen. The results show high concentrations of lead in the thalles for the three species, indicating high pollution by lead in this city. In order to appreciate the spatial distribution leaving this pollution and its environmental impact in urban areas, it was developed pollution maps based on the results of each species.
\end{abstract}

Keywords: Traffic, pollution, lead, mosses, lichens, mapping, Tlemcen, Algeria

\section{Résumé}

L'évaluation de l'intensité de la pollution, via l'accumulation d'éléments polluants de l'atmosphère, par les organismes vivants constitue 
une technique environnementale très efficace utilisée depuis plusieurs décennies. La mesure de l'accumulation dans ces organismes permet de pallier les difficultés rencontrées lors des mesures physico-chimiques directes, souvent très complexes et couteuses.

Par leurs caractéristiques physiologiques et morphologiques, les mousses et les lichens s'avèrent très efficaces pour répondre à cette problématique, notamment leur capacité à accumuler les contaminants en l'occurrence les éléments traces métalliques tels que le plomb.

L'objectif de ce travail consiste à mesurer les concentrations en plomb d'origine routière dans les différentes entités urbaines de la ville de Tlemcen, et sa périphérie en utilisant une espèce de mousse et deux espèces de lichens. Les résultats obtenus montrent de fortes teneurs en plomb dans les thalles des trois espèces, ce qui traduit un fort taux de pollution au niveau de ville. Afin d'apprécier la répartition spatiale de cette pollution et son impact environnemental en milieu urbain, il a été élaborée des cartes de pollution en fonction des résultats de chaque espèce.

Mots-clés : Trafic routier, pollution, plomb, mousses, lichens, cartographie, Tlemcen, Algérie

\section{Introduction}

La Biosurveillance de la qualité de l'air est un outil simple et peu couteux, complémentaire des mesures physico-chimiques réalisées par les différents réseaux de surveillance. Elle ne permet pas de connaitre la concentration de l'air en polluants, mais elle donne des indications assez précises sur les impacts potentiels que ces polluants atmosphériques peuvent avoir sur les êtres vivants.

De nombreux végétaux peuvent être utilisés pour la biosurveillance de la qualité de l'air, soit parce qu'ils présentent des réactions biologiques caractéristiques en réponse à certains polluants, soit parce qu'ils accumulent dans leurs tissus certains des composés toxiques qui se trouvent dans l'air qui les entoure. Les organismes vivants sont connus pour témoigner des conditions environnementales selon leur sensibilité. Il s'agit de surveillance biologique, en utilisant les espèces animales ou végétales les plus sensibles face aux polluants recherchés dans leur milieu environnant. Dans ce contexte, les lichens et les mousses sont d'excellents bioindicateurs pour évaluer la pollution atmosphérique (Garrec et van Haluwyn, 2002). Ils permettent une surveillance environnementale à proximité des sources fixes notamment les écosystèmes urbains.

Pour bien illustrer ces aspects, plusieurs approches ont été proposées selon l'échelle d'observation considérée (Markert et al., 2003). On distingue ainsi l'échelle écologique par la réponse biologique des individus 
(bioindication) ou des communautés (biointégration) et l'échelle géochimique par l'accumulation de contaminant (bioaccumulation).

L'utilisation des mousses et des lichens comme bioaccumulateurs d'éléments traces métalliques a donné des résultats impressionnants au cours des 40 dernières années dans différentes parties du monde au vue des différents travaux réalisés (Rühling, et all., 1969, Tyler, 1990, Grodzinska et all., 1990, Thoni et all.,1996, Gerdoll et all., 2000, Fernandez et all.,2002, Garrec,2002, Malandrino et all., 2006).

Pour le cas de l'Algérie, des travaux scientifiques ont été réalisés pour déterminer les concentrations des substances chimiques nocives pour les végétaux des milieux urbains. Ainsi, plusieurs chercheurs ont démontré que les plantes et les animaux sensibles, peuvent être de véritables bioindicateurs de pollution. L'utilisation de la bioindication s'avère très utiles dans les agglomérations urbaines réputées à forte pollution. Les approches appliquées ont consisté à doser les éléments traces métalliques $(\mathrm{Pb}, \mathrm{Cd} \mathrm{Zn}, \mathrm{Cu}$, etc.) dans les thalles du lichen Xanthoria parietina dans différents sites de quelques agglomérations algériennes (Samadi, 1993; Rahali, 2003; Maatoug, 2010).

L'objectif de ce travail consiste à évaluer les concentrations en plomb d'origine routière et d'établir une cartographie de la pollution à l'aide d'une espèce de mousses Bryum argenteum et deux espèces de lichens foliacés, Xanthoria parietina et Parmelia sulcata transplantées dans divers sites du périmètre urbain de la ville de Tlemcen.

\section{Présentation de la zone d'étude}

La wilaya de Tlemcen est située à l'extrême Nord-ouest du pays. Sa position géographique et son niveau d'équipement et d'infrastructures, lui confèrent un rôle de carrefour stratégique et très dynamique dans les échanges Est-Ouest et Nord-Sud superficie $9200 \mathrm{~km}^{2}$

(figure $\mathrm{N}^{\circ} 1$ ). Elle s'étend sur une

La ville de Tlemcen occupe la partie centrale de la wilaya. Elle regroupe une population de 250000 habitants soit près de $20 \%$ de la population totale. Son tissu urbain s'étend sur près de 1000 hectares. Le relief est dans l'ensemble accidenté et contrasté. Les pentes sont très abruptes. Les altitudes varient entre 600 et $1025 \mathrm{~m}$.

Le climat est de type méditerranéen avec un régime pluviométrique variable laissant apparaitre deux saisons bien distinctes l'une pluvieuse qui s'étend de septembre à avril, et l'autre chaude et sèche. Ce ci exprime le contraste très important qui sévit durant l'année. La moyenne des précipitations oscille entre 500 et $550 \mathrm{~mm} / \mathrm{an}$. L'année la plus arrosée est celle de 1950 avec $823 \mathrm{~mm}$ par ailleurs, l'année la plus sèche est celle de 1983 avec seulement $250 \mathrm{~mm}$. 
Les températures enregistrent également des fluctuations importantes avec un fort écart thermique entre l'été et l'hiver. Le mois le plus froid est février avec $4^{\circ} \mathrm{C}$ et le mois le plus chaud est aout avec $36{ }^{\circ} \mathrm{C}$.

Les vents dominant sont ceux du nord-ouest en hiver en raison de l'influence maritime. Ils sont généralement très froids et humides. La saison sèche est caractérisée par des vents chauds et secs provenant des zones sud (Sirocco).

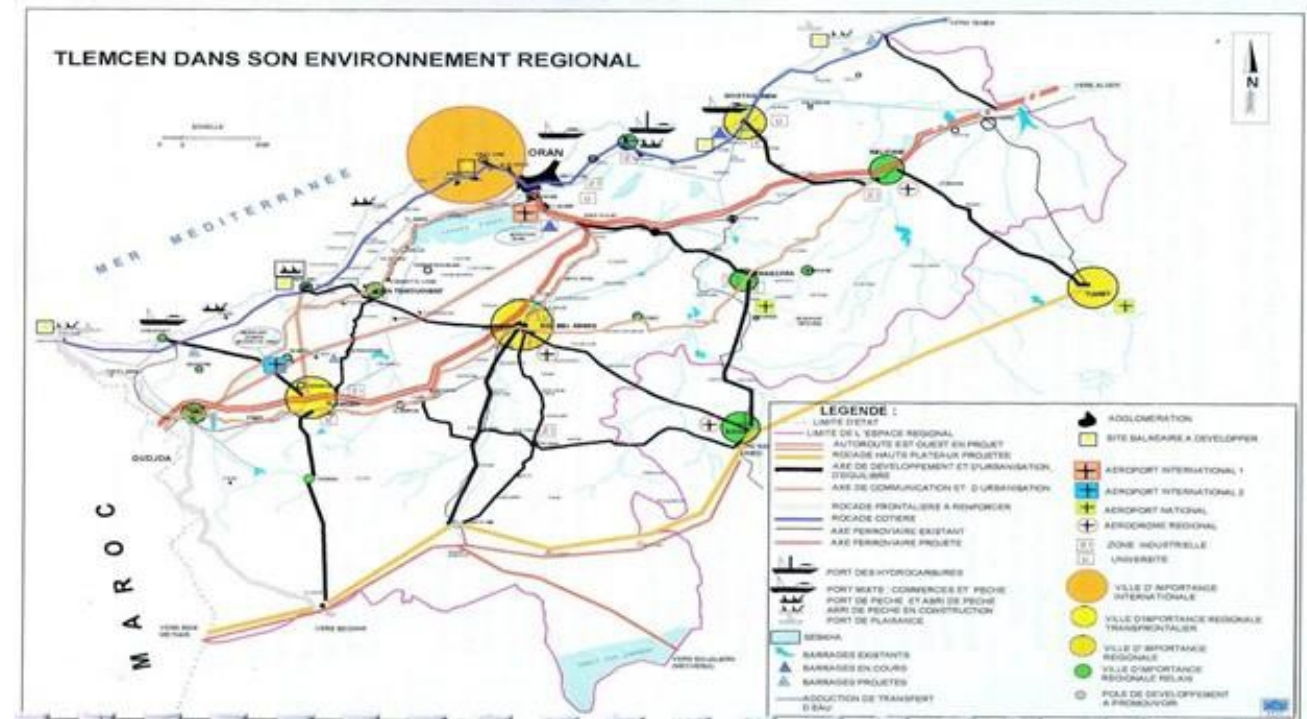

Figure $\mathrm{N}^{\circ} 1$ : Tlemcen dans son ensemble régional (ANAT Tlemcen, 2014)

\section{Le Réseau Routier et Parc Automobile :}

En raison de sa position frontalière, Tlemcen est desservi par un réseau routier très dense lui facilitant les échanges avec le reste du territoire. Il s'étend sur un linéaire de $542 \mathrm{~km}$ (DTP, 2014). Il est représenté en grande partir par le tronçon de l'autoroute est-ouest, la RN 35 (Tlemcen Maghnia), et la RN 7 (Tlemcen - Sidi Bel Abbes) pour les échanges estouest, et la RN 22 (Tlemcen - Sebou), pour les échanges nord sud. Ce réseau présente dans l'ensemble des caractéristiques géométriques et un état jugé relativement satisfaisant. Ces axes assurent une intensité de trafic très intense confirmée par les opérations de comptage de trafic automobile. La densité du réseau routier total est de $5.2 \mathrm{~km} / \mathrm{km}^{2}$.

Le parc automobile est estimé à 253527 véhicules tous types confondus (service automobile, 2014). Il présente une forte hétérogénéité tant sur le plan type et âge des véhicules et la nature du carburant. Les véhicules de type léger représentent environ $55 \%$ du total. Concernant la nature du carburant, les voitures diesel continuent toujours à constituer une part non négligeable du total avec un taux de $41.6 \%$. Cet état de fait est encouragé par des prix relativement compétitifs du gasoil par rapport à 
l'essence. L'usage du GPL demeure encore faible malgré de multiples compagnes de vulgarisation et de sensibilisation pour la généralisation de l'usage des énergies propres.

Pour ce qui est de la répartition des véhicules par structure d'âge, on remarque une forte tendance à son rajeunissement durant les dix dernières années. Les véhicules de moins de cinq ans occupent environ $32 \%$ du parc total. Néanmoins les statistiques de l'année 2014, du service automobile, montrent que les véhicules âgés de plus de dix ans occupent encore près de $59 \%$ du total.

\section{Technique et démarche de transplantation}

Les mousses et lichens transplantés ont été prélevés au niveau du Parc National de Tlemcen. Il présente dans l'ensemble les mêmes conditions écologiques que la zone d'étude. Les prélèvements ont été effectués dans les zones les plus reculées du parc et loin des voies de communication pour éviter toute source de pollution. Les échantillons ont été récoltés sur troncs d'arbres pour s'affranchir de l'influence du sol. Le choix de travailler sur des espèces corticoles se justifie également par la volonté de s'éloigner au maximum du sol, environnant pouvant potentiellement influencer la composition de l'échantillon, notamment en métaux (Bargagli et Nimis, 2002).

Les mousses et les lichens sont reconnus comme étant de très bons bioindicateurs et bioaccumulateurs de la pollution notamment en éléments trace métallique tels que le plomb, le zinc, le cuivre, le cadmium et le mercure. (Biziuk, 2003 ; Cassagne et all 2007).

Les transplantations ont été effectuées sur environ 80 sites en procédant à un maillage systématique de l'agglomération en vue de cerner toute la zone d'étude. Les mailles ont des dimensions de $(0.5 \times 0.5 \mathrm{~km})$ selon Garrec et Van Haluwyn (2002).

\section{Résultats et discussions}

\section{Mousse : Bryum argenteum}

Le dosage du plomb a été effectué sur 38 échantillons. On a procédé à regrouper les stations dont les concentrations se rapprochent fortement. Pour ce faire, on a opté pour l'application du test de la classification automatique. Les résultats obtenus sont illustrés dans la figure $\mathrm{N}^{\circ} 2$. A première vue, quatre classes de pollutions sont mises en évidence. 


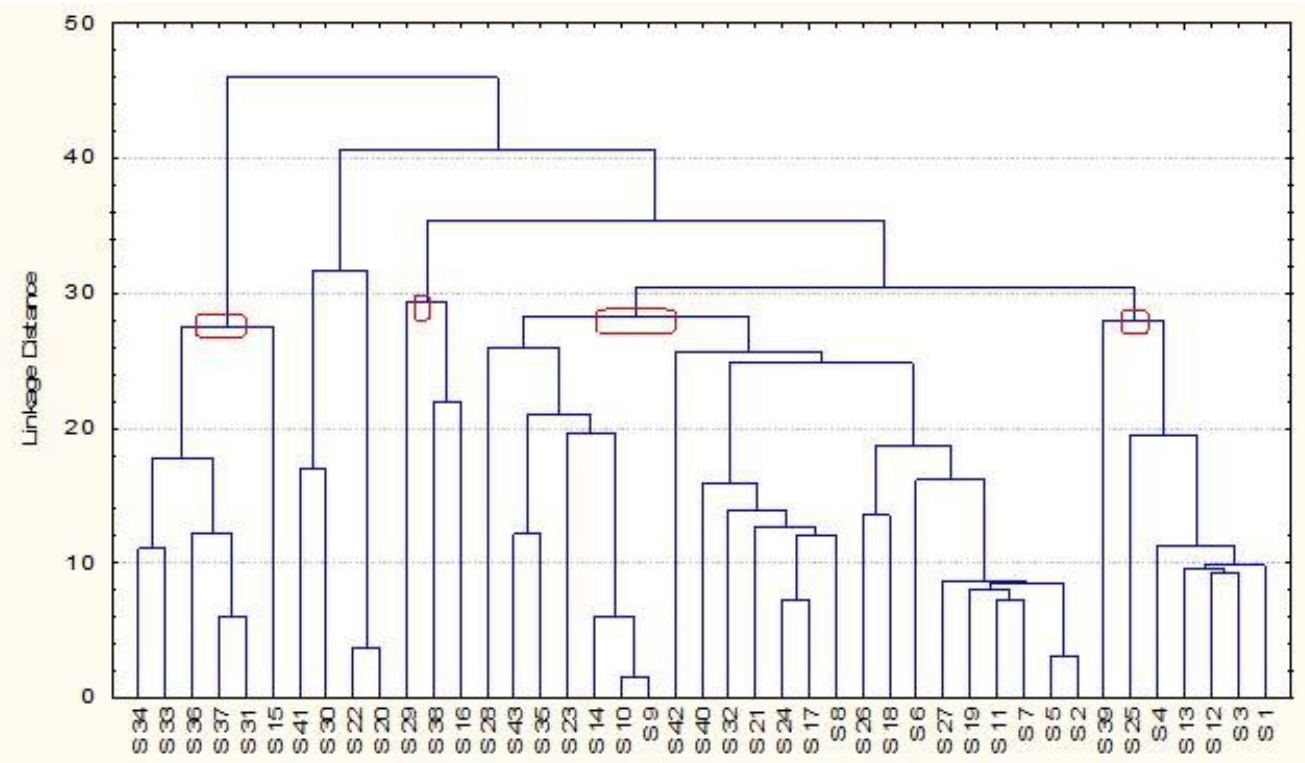

Figure $\mathrm{N}^{\circ} 2$ : Classification automatique des données dans les différentes stations de la ville de Tlemcen (cas de Mousse : Bryum argenteum)

L'examen du tableau $\mathrm{N}^{\circ} 1$ permet de constater que les teneurs moyennes en plomb varient entre $206,81 \pm 18,26 \mu \mathrm{g} / \mathrm{g}$ et $646,50 \pm 16,94$ $\mu \mathrm{g} / \mathrm{g}$. Ces valeurs sont nettement très supérieures aux valeurs des témoins $(15.46 \pm 0.68 \mu \mathrm{g} / \mathrm{g})$.

Les fortes concentrations sont enregistrées au niveau de la classe $\mathrm{N}^{\circ} 4$ avec une moyenne de $646 \mu \mathrm{g} / \mathrm{g}$ ) et une valeur extrême de l'ordre de 678,48 $\mu \mathrm{g} / \mathrm{g}$. Cette classe appelée communément classe chaude regroupe les sites les plus pollués de la ville notamment le centre-ville qui connait un trafic automobile très intense. Cet état de fait est dû à une forte concentration des équipements générateurs de trafic (commerces et administrations). L'effet de la pente et les remontées de file augmente l'intensité du freinage. A cela, s'ajoutent d'autres lieux tels que l'échangeur de l'autoroute est - ouest et ceux de la mini rocade qui désaffectent le trafic nord - sud et est - ouest.

Les concentrations moyennes sont observées au niveau des classes 3 avec des valeurs de 521,52 $\pm 28,16 \mu \mathrm{g} / \mathrm{g}$. Cette classe représente les entités mitoyennes au centre-ville.

Les classes 2 et 1 regroupent quant à elles les concentrations relativement faibles avec des concentrations respectives de 388,23 $\pm 16,42$ $\mu \mathrm{g} / \mathrm{g}$ et $206,81 \pm 18,26 \mu \mathrm{g} / \mathrm{g}$. Ces deux dernières caractérisent les sites où les émissions sont généralement faibles à moyennes. Ceci est dû :

- d'une part à une circulation moindre comparativement au centreville ; 
- d'autre part, ces entités périphériques sont plus ou moins ouvertes et connaissent une urbanisation moins dense. De ce fait la circulation des masses d'air favorise la dispersion des polluants.

Les zones de la classe 2 et 1 sont représentées par les zones de Boudjlida, Bouhennak, Koudia, Saf Saf, Chetouane, Abou Tachfine etc.

Tableau $\mathrm{N}^{\circ} 1$ Statistique descriptive des classes de pollution en $(\mu \mathrm{g} / \mathrm{g})$ selon la classification automatique

\begin{tabular}{|l|c|c|c|c|c|c|c|c|}
\hline & $\mathrm{N}$ & Moyenne & Médiane & Minimum & Maximum & $1^{\text {er }}$ Quartile & 3eme Quartile & $\begin{array}{c}\text { Ecart } \\
\text { type }\end{array}$ \\
\hline Classe 1 & 5 & 206,81 & 211,15 & 182,51 & 223,45 & 193,57 & 223,35 & 18,26 \\
\hline Classe 2 & 3 & 388,23 & 397,24 & 369,27 & 398,19 & 369,27 & 398,19 & 16,42 \\
\hline Classe 3 & 23 & 521,52 & 540,51 & 432,62 & 601,27 & 452,94 & 564,91 & 28,16 \\
\hline Classe 4 & 7 & 646,50 & 641,21 & 629,27 & 678,48 & 631,54 & 654,18 & 16,94 \\
\hline Témoins & 3 & 15,46 & 15,17 & 14,98 & 16,25 & 14,98 & 16,25 & 0,68 \\
\hline
\end{tabular}

D'une manière générale, on peut dire que les mousses absorbent directement l'eau et les sels nutritifs ainsi que les polluants présents dans les dépôts atmosphériques. L'absence de système racinaire ainsi qu'une cuticule bien développé minimise l'influence du substrat sur les niveaux de contaminants dans leurs tissus (Rzepka et Cuny, 2008).

L'accumulation se fait principalement par des mécanismes d'échanges cationiques avec une très faible résistance à la diffusion des éléments absorbés.

Les résultats obtenus sont en parfaite adéquation avec des travaux similaires réalisés avec des mousses notamment ceux de Bargagli et all (1998; 2002).

\section{Les lichens}

\section{Xanthoria parietina}

La classification automatique des 43 échantillons dosés a permis de dégager trois classes bien distinctes. Les classes 2 et 3 rassemblent les sites les plus pollués. Par ailleurs, la classe 1 regroupe les sites où la pollution est moins ressentie.

Les fortes concentrations en plomb sont donc enregistrées au niveau de la classe 3 avec une moyenne de l'ordre de $1137.59 \pm 11.47 \mu \mathrm{g} / \mathrm{g}$. 


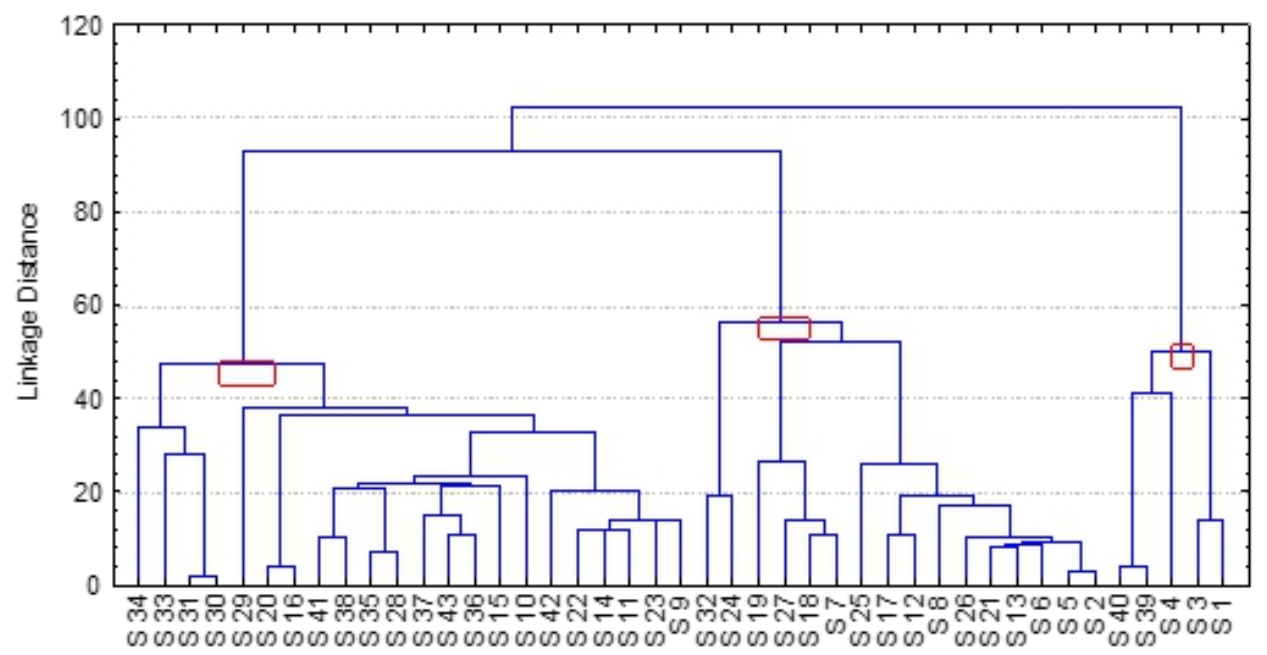

Figure $\mathrm{N}^{\circ} 3$ : Classification automatique des données dans les différentes stations de la ville de Tlemcen

Cette classe regroupe les sites les plus pollués notamment le centre villes et les grandes artères qui concentrent les principaux équipements. Cette situation est d'autant plus accentuée par la topographie très accidentée du site de l'agglomération. La classe 2 laisse apparaitre beaucoup de sites (16 échantillons) avec des émissions de plomb relativement moindre. La moyenne obtenue est de l'ordre de $855.21 \pm 22,49 \mu \mathrm{g} / \mathrm{g}$.

La classe 1 quant à elle avec une moyenne de $472,52 \pm 13.5 \mu \mathrm{g} / \mathrm{g}$ affiche néanmoins des concentrations fortement inférieures aux deux classes précédentes. Toutefois ces valeurs restent fortement supérieures aux valeurs des échantillons témoins (tableau $\mathrm{N}^{\circ} 2$ ). Les résultats montrent que même les quartiers situés en retrait par rapport aux grandes voies de communication sont affectés par la pollution du plomb d'origine routière.

Tableau $\mathrm{N}^{\circ} 2$ Statistique descriptive des classes de pollution en $(\mu \mathrm{g} / \mathrm{g})$ selon la classification automatique

\begin{tabular}{|l|c|c|c|c|c|c|c|c|}
\hline & $\mathrm{N}$ & Moyenne & Médiane & Minimum & Maximum & $1^{\text {er }}$ Quartile & $\begin{array}{c}\text { 3eme } \\
\text { Quartile }\end{array}$ & $\begin{array}{c}\text { Ecart } \\
\text { type }\end{array}$ \\
\hline classe 1 & 22 & 472.52 & 563,001 & 378.96 & 568.92 & 689,14 & 524,26 & 13,15 \\
\hline classe 2 & 16 & 855.21 & 923,65 & 780.56 & 958.22 & 1022,23 & 859,67 & 22,49 \\
\hline classe 3 & 5 & 1137.59 & 1156,04 & 1122.23 & 1145.56 & 1192,13 & 1141,81 & 46,47 \\
\hline Témoins & & 17.85 & 19,27 & 17.25 & 19.44 & 21,14 & 17,25 & 21,14 \\
\hline
\end{tabular}

\section{Parmelia sulcata}

Les résultats obtenus avec l'espèce Parmelia sulcata confirment parfaitement les résultats obtenus avec les mousses et le lichen Xanthoria 
parietina. Les concentrations sont de loin supérieures aux valeurs des échantillons témoins.

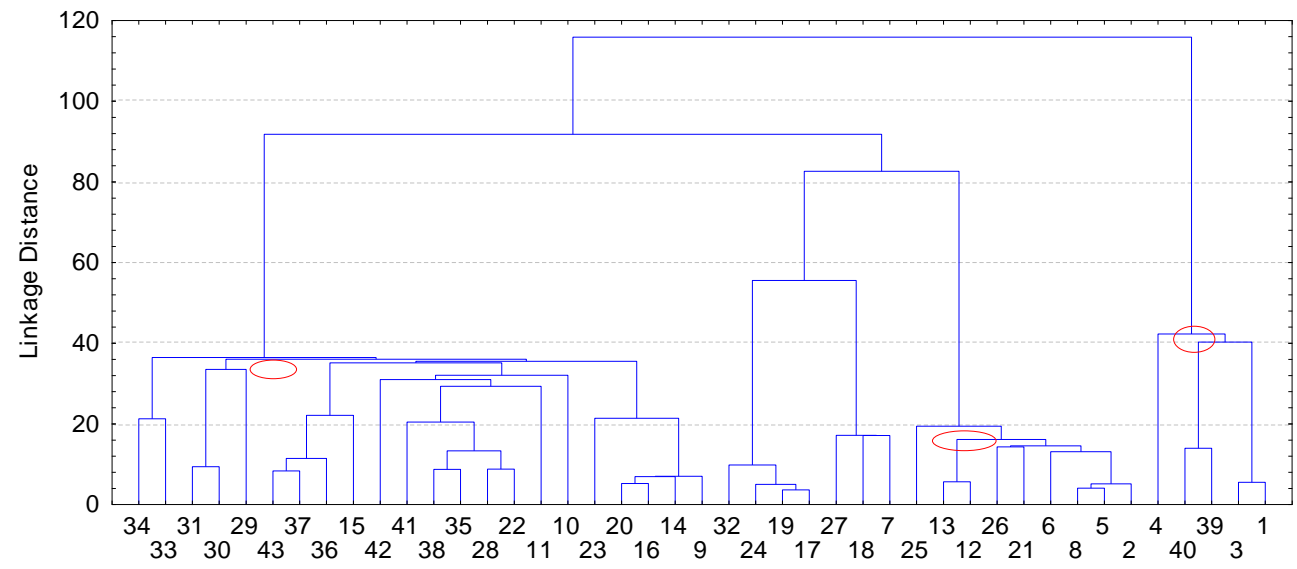

Figure $\mathrm{N}^{\circ} 4$ : Classification automatique des données dans les différentes stations de la ville de Tlemcen

La classification automatique des 34 échantillons dosés a permis de ressortir 3 groupes de classes bien distinctes.

La classe 3 regroupe toujours les sites les plus pollués qui sont affectés d'un trafic intense (tableau $\mathrm{N}^{\circ} 3$ ). La moyenne obtenue est de l'ordre de $1165,58 \pm 19,47 \mu \mathrm{g} / \mathrm{g}$.

Tableau $\mathrm{N}^{\circ} 3$ Statistique descriptive des classes de pollution en $(\mu \mathrm{g} / \mathrm{g})$ selon la classification automatique

\begin{tabular}{|c|c|c|c|c|c|c|c|c|}
\hline & $\mathrm{N}$ & Moyenne & Médiane & Minimum & Maximum & $1^{\text {er }}$ Quartile & $\begin{array}{c}\text { 3eme } \\
\text { Quartile }\end{array}$ & $\begin{array}{c}\text { Ecart } \\
\text { type }\end{array}$ \\
\hline Classe 1 & 22 & 583,17 & 591,89 & 396,86 & 715,28 & 532,47 & 653,01 & 15.22 \\
\hline Classe 2 & 9 & 984,61 & 982,62 & 965,34 & 1017,22 & 969,53 & 997,19 & 13.25 \\
\hline Classe 3 & 5 & 1165,58 & 1167,33 & 1131,19 & 1190,58 & 1153,41 & 1185,41 & 19.47 \\
\hline Témoins & 3 & 19,26 & 18,21 & 16,14 & 23,45 & 16,14 & 23,45 & 1.58 \\
\hline
\end{tabular}

La classe 2 réunit les sites où les émissions sont moindres. Elle se caractérise par une moyenne de 984,61 $\pm 13,25 \mu \mathrm{g} / \mathrm{g}$. Les faibles concentrations sont regroupées au niveau de la classe 1 avec une moyenne de $583,17 \pm 15,22 \mu \mathrm{g} / \mathrm{g}$.

\section{Cartographie}

Les résultats obtenus ont été utilisés pour l'élaboration d'une série de cartes de pollution pour chaque espèce. Cette approche permet de mieux appréhender la répartition des différents niveaux de pollution sur le plan spatial et de les comparer avec l'intensité du trafic supporté par chaque site. 


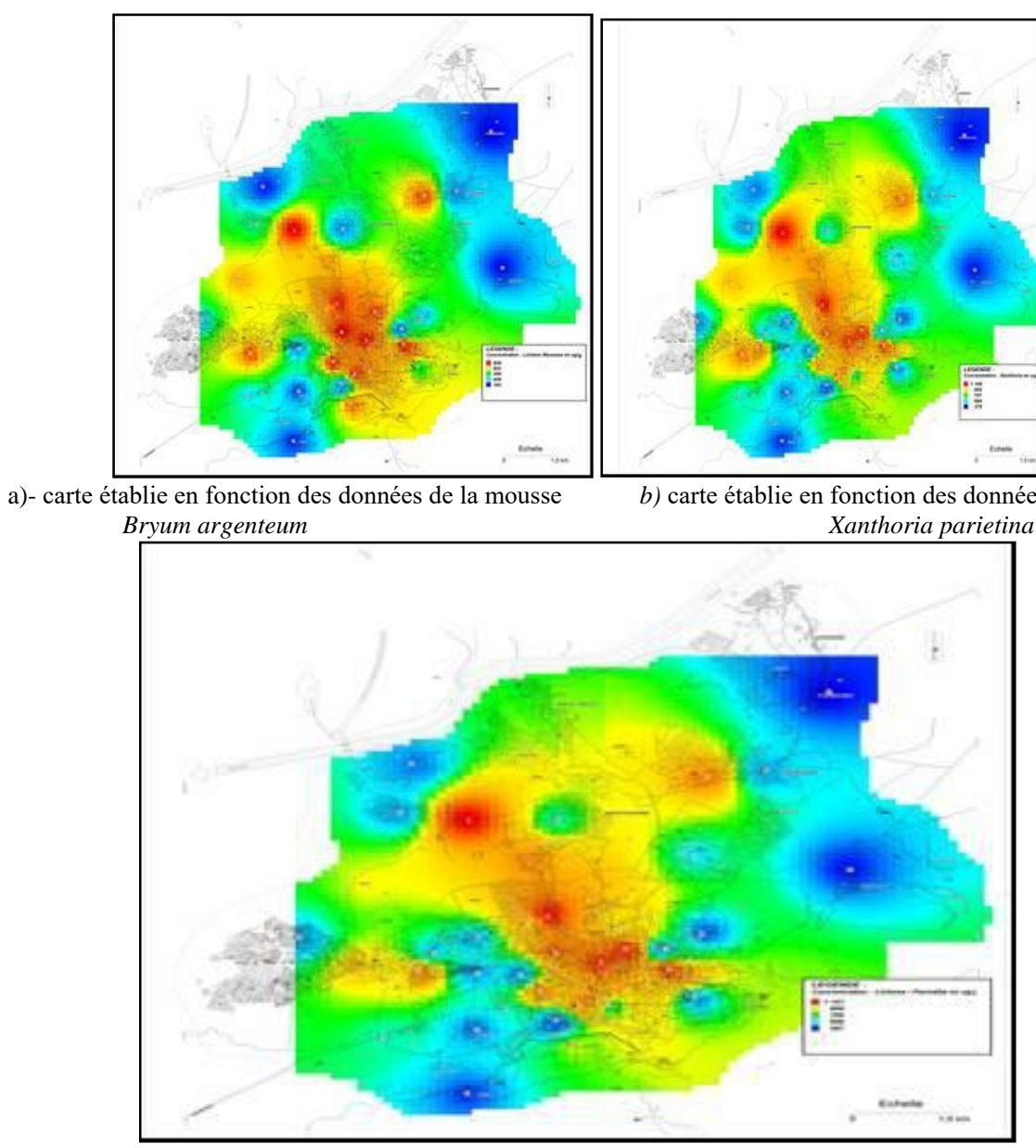

c) carte établie en fonction des données du lichen Parmelia sulcata

Figure $\mathrm{N}^{\circ} 5$ : Carte de pollution atmosphérique par le plomb d'origine routière de la ville de Tlemcen

La lecture des trois cartes permet de mettre en évidence la dominance de la couleur rouge au niveau du centre-ville et la zone nord qui structure la totalité du trafic est - ouest et nord - sud (figure $\mathrm{N}^{\circ} 5$ ). Les concentrations en plomb sont très fortes et dépassent largement les $1000 \mu \mathrm{g} / \mathrm{g}$. Ces zones devront faire l'objet d'une attention particulière par la création éventuelle d'espaces piétons. La configuration spatiale du centre-ville avec la prédominance de la médina (ancienne ville précoloniale) avec des voies très étroites laissent très peu de chance aux gaz d'échappement de se disperser dans l'atmosphère.

D'un autre coté la zone nord doit être soulagée par le report d'une partie du trafic vers d'autres pénétrantes ou la réalisation de nouvelles infrastructures routières pour bien organiser et canaliser le trafic de transit et faciliter par conséquent, l'accès au centre-ville. 
La couleur jaune affecte plus de $30 \%$ de la superficie urbanisée. Elle manifeste une tendance pessimiste quant à l'évolution du niveau de pollution par le plomb. Elle est représentée par les zones attenantes au centre-ville et les nouvelles concentrations d'habitat. Les valeurs oscillent entre 800 et $1000 \mu \mathrm{g} / \mathrm{g}$. Ces zones sont représentées par les quartiers des oliviers, Sidi Said, Kiffane, Imama qui permettent un accès direct et rapide au centre-ville.

La couleur verte caractérise les zones est et ouest de l'agglomération de Tlemcen. Les concentrations varient entre 700 et $800 \mu \mathrm{g} / \mathrm{g}$. Elles sont représentées par les entités de Chetouane, Agadir, Bouhennak, Hai El Waai, Boudjlida et Ouzidane.

La couleur bleu ciel concerne les zones situées au sud, à l'ouest et à l'est de la ville. Les concentrations varient entre 500 et $700 \mu \mathrm{g} / \mathrm{g}$. Il s'agit notamment des quartiers de :

Boudghene et Birouana (sud de la ville), quartiers résidentiels sur les hauteurs de la ville connaissant très peu de trafic ;

Agadir et Sidi Boumediene, quartiers résidentiels avec un accès relativement difficile ;

- Imama et Kiffane ouest, quartiers résidentiels par excellence. De plus la prédominance d'un type d'habitat peu dense (individuel et semi collectif) ainsi que des voies très larges facilitent la dispersion rapide des polluants.

La dernière classe représentée par la couleur bleu se caractérise par une pollution relativement faible. Elle concerne les entités qui connaissent une faible urbanisation. De plus, elles sont situées en retrait par rapport aux grandes voies de communication. On citera à titre d'exemple le quartier de Saf Saf.

\section{Conclusion}

La présente étude a pour objectif la cartographie et l'estimation des niveaux de pollution par le plomb d'origine routière à l'aide d'une espèce de mousse bryum argenteum et deux espèces de lichens, Xanthoria parietina et Parmelia sulcata, transplantées dans divers sites de la ville de Tlemcen.

Les résultats obtenus ont permis de mis en évidence la présence d'une réelle pollution qui affecte le centre-ville et sa périphérie immédiate. Les valeurs moyennes en plomb oscillent entre 800 et $1100 \mu \mathrm{g} / \mathrm{g}$.

Les zones périphériques sont également affectées par la pollution, mais avec des valeurs relativement moindres comparativement à celles du centre-ville. 600 à $800 \mu \mathrm{g} / \mathrm{g}$. Ceci confirme l'affinité élevée des lichens et des mousses d'accumuler les polluants atmosphériques, avec une légère prédominance pour les mousses. Elles sont également en parfaite adéquation avec d'autres travaux similaires réalisées dans d'autres villes en Algérie notamment la ville de Tiaret. 
L'approche cartographique a permis de spatialiser les niveaux de pollution et les variations des concentrations en plomb dans les différents quartiers de la ville.

L'accroissement du niveau de pollution au niveau de la ville est dû en grande partie à l'accroissement et le vieillissement du parc automobile. De ce fait, une attention particulière doit être accordée à cet aspect en vue de le ramener à des seuils tolérables par l'adoption de certaines mesures notamment :

- $\quad$ La maitrise du parc automobile ;

- La réduction des émissions des polluants par l'encouragement de l'usage de filtres appropriés ;

- $\quad$ L'usage de carburants moins polluants tel que le GPL.

\section{References:}

Agence Nationale d'Aménagement du Territoire (ANAT) ; Etude du Plan directeur d'aménagement et d'urbanisme de la commune de Tlemcen (2014), page 147.

Bargagli, R; D'Amato,F; Zonation of trace metal accumulation in three species of epiphytic lichens belonging to the genus Parmelia. Cryptogamie. Bryologie, Lichenologie, (1987), vol. 8, nº4, pp. 331-337.

Bargagli, R; Trace elements in terrestrial plants. An ecophysiological approach to biomonitoring and

Bargagli, R ; Monaci, F ; Borghini, F ; Bravi, F ; Agnorelli, C. Mosses and lichens as biomonitors of trace metals. A comparison study on Hypnum cupressiforme and Parmelia caperata in a former mining district in Italy. Environnemental Pollution 116 (2002) 279 - 287.

Biziuk, M ; Aspects of the biomonitoring studies using mosses and lichens as indicators of metal pollution. Environmental Research. Volume 93, Issue 3, November 2003, Pages 221-230

Conti, M ; Cecchetti, G ; Biological monitoring : lichens as bioindicators of air pollution assessment. Environmental Pollution; Volume 114, Issue 3, October 2001, Pages 471-492.

Garrec J.-P., Van Haluwyn C. Biosurveillance végétale de la qualité de l'air. Concepts, méthodes et applications. Éditions Tec \& Doc, Lavoisier, Paris $2002: 118$ p.

Loppi , S ; Ivanov, D ; Boccardi, R ; Biodiversity of epiphytic lichens and air pollution in the town of Siena (Central Italy). Environmental pollution, $2002 ; 11-117$.

Maatoug M., Medkour K., Ait Hammou M., Hellal B. Cartographie de la pollution atmosphérique par le plomb d'origine routière à l'aide de transplantation d'un lichen bio-accumulateur Xanthoria parietina dans la ville de Tiaret (Algérie). Pollution atmosphérique 2010 ; 205 : 93-101. 
Malandrino, M ; Adsorption of heavy metals on vermiculite : Influence of $\mathrm{pH}$ and organic ligands. Journal of Colloid and Interface Science. Volume 299, Issue 2, 15 July 2006, Pages 537-46

Markert, B ; A comparison of heavy metal deposition in selected Eastern European countries using the moss monitoring method, with special emphasis on the 'Black Triangle'. Science of The Total Environment. Volume 193, Issue 2, 20 December 1996, Pages 85-100.

Rühling, K ; Sorption and Retention of Heavy Metals in the Woodland Moss Hylocomium splendens. Åke Rühling and Germund Tyler Oikos. Vol. 21, No. 1 (1970), pp. 92-97.

Rzepka, M-A ; Mécanismes d'accumulation et de toxicité des composés organiques volatils chez les végétaux supérieurs. Thèse de doctorat de l'université de Lille II, soutenue en janvier 2008. 248 p.

Semadi, A ; Deruelle, S; Détection de la pollution plombique à l'aide de transplants lichéniques dans la région d'Annaba (Algérie). Pollution atmosphérique Octobre-Décembre 1993 : 86-101.

Service automobile de la wilaya de Tlemcen; bureau des cartes grise, données statistiques, décembre 2014. 